\title{
Análisis cariotípicos en dos especies de Halerpestes (Ranunculaceae) del Noroeste Argentino
}

\author{
Karyotypic analysis of two species of Halerpestes (Ranunculaceae) \\ from Argentine Northwest
}

Páez, Valeria de los A.*; Aldo R. Andrada

Instituto de Genética, Fundación Miguel Lillo. Miguel Lillo 251, (T4000JFE) Tucumán, Argentina.

* Autor corresponsal: paezvaleria@hotmail.com

\section{RESUMEN}

Halerpestes (Ranunculaceae) es un género pequeño el cual contiene 13 especies. Tres de ellas son típicas de la región del Noroeste Argentino: $H$. cymbalaria, $H$. exilis y $H$. uniflora. En investigaciones recientes se separó a $H$. exilis como una especie diferente de $H$. cymbalaria, ya que era considerada una forma de esta última. En este trabajo se realizaron recuentos cromosómicos y cariotipos de $H$. cymbalaria y $H$. exilis mediante técnicas citogenéticas clásicas. Los resultados fueron concluyentes en que ambos taxones son diploides y presentan $2 \mathrm{n}=16$ cromosomas, cariotipos asimétricos y unimodales; sin embargo, existen diferencias en el tamaño cromosómico y la organización de la cromatina, teniendo $H$. exilis un par cromosómico con satélite no observado en el otro taxón. La ubicación de $H$. cymbalaria y $H$. exilis como especies diferentes, realizada por diferentes autores, es afianzada por nuestros resultados.

Palabras clave - Cariotipo, mitosis, nivel de ploidía.

\section{ABSTRACT}

Halerpestes (Ranunculaceae) is a small genus with 13 species. Three of them are typical from the Northwest region of Argentina: H. cymbalaria, H. exilis and H. uni-

\footnotetext{
> Ref. bibliográfica: Páez, V. de los A.; Andrada, A. R. 2019. "Análisis cariotípicos en dos especies de Halerpestes (Ranunculaceae) del Noroeste Argentino". Lilloa 56 (2): 59-66. Fundación Miguel Lillo, Tucumán, Argentina. D.O.I.: doi.org/10.30550/j.lil/2019.56.2/5

> Recibido: 07/03/19 - Aceptado: 08/08/19

$>$ URL de la revista: http://lilloa.lillo.org.ar

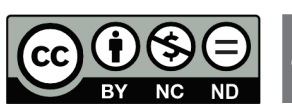

> Algunos derechos reservados. Esta obra está bajo una Licencia Creative Commons Atribución - No Comercial - Sin Obra Derivada 4.0 Internacional.
} 
flora. In recent investigations, $H$. exilis was separated as a different species than $H$. cymbalaria, since it was considered as a variety of the last species. In this work, chromosomal counts and karyotypes of $H$. cymbalaria and $H$. exilis were performed using classical cytogenetic techniques. The results were conclusive in that both taxa are diploid and present $2 \mathrm{n}=16$ chromosomes, asymmetric and unimodal karyotypes; however, there are differences in chromosomal size and chromatin organization, with H. exilis having a chromosomal pair with satellite not observed in the other taxon. The taxonomic location of $H$. cymbalaria and $H$. exilis as different species, carried out by different authors is reinforced by our results.

Keywords - Karyotype, mitosis, ploidy level.

\section{INTRODUCCIÓN}

La familia Ranunculaceae contiene 2500 especies en 59 géneros, de distribución cosmopolita, la mayoría de las especies que la integran crecen en regiones templadas y frías; el resto de sus taxones se encuentran en regiones montañosas tropicales y subtropicales (Ziman y Kenner, 1989).

Greene (1899) propuso el género Halerpestes Greene, en base al modo de propagación de la planta a través de estolones que enraizan en sus articulaciones para producir nuevas plantas, a la textura delgada de sus frutos y a la superficie estriada de sus aquenios. Las mencionadas características permitieron reubicar dentro de este género taxones que pertenecían a Ranunculus L., Oxygraphis Bunge y Cyrtorhyncha Nutt. (Greene, 1899). El género Halerpestes ha sido recientemente confirmado a través de estudios de su morfología, hábitat y datos moleculares realizados por Edmadzade, Lehnebach, Lockhart, Hörandl (2010).

Halerpestes, reúne 13 especies según los datos de Trópicos (http://www.tropicos.org), tres de ellas crecen en Argentina: H. cymbalaria (Pursh) Greene, H. exilis (Phil.) Tamura y H. uniflora (Phil. ex Reiche) Emadzade, Lehnebach, P.J. Lockh. \& Hörandl (Lourteig, 1951; Emadzade et al., 2010). H. cymbalaria y H. exilis se citaron como una misma especie bajo el nombre de Ranunculus cymbalaria Pursh. (Zuloaga y Morrone, 1999), pero posteriormente fueron mencionadas nuevamente como especies diferentes dentro del género Halerpestes (Zuloaga, Morrone y Belgrano, 2008; Emadzade et al., 2010).

Halerpestes cymbalaria es una hierba perenne, nativa, que se distribuye entre los 0-4500 m snm en las regiones del Noroeste Argentino, Cuyo, Centro y Sur de Argentina, mientras que $H$. exilis es un taxón anual, nativo, que se encuentra entre los 2000-4300 m snm en las regiones NOA y Cuyo (Zuloaga et al., 2008). Morfológicamente se diferencian entre ellas por el porte de la planta y la forma de las hojas, en Halerpestes cymbalaria la planta puede alcanzar un tamaño de 10 a $25 \mathrm{~cm}$, con lámina generalmente suborbicular, ovoda, rara vez trilobada, mientras que en $H$. exilis el tamaño de planta varía de 1 a $3,5 \mathrm{~cm}$, hojas profundamente trilobadas hasta casi partidas y lóbulos o segmentos laterales a menudo subdivididos (Lourteig, 1951; Kiesling, 1994). 
Ambos taxones suelen encontrarse en lugares muy húmedos, en banquinas anegadas, próximas a arroyos y vertientes (Lourteing, 1951; Lozzia, 2007).

Los estudios citogenéticos de la familia Ranunculaceae llevados a cabo por Langlet (1932) y Gregory (1941) se basaron en el tamaño de los cromosomas y en los números cromosómicos básicos, permitiendo agrupar a las especies en dos grandes grupos con diferentes tipos de cromosomas denominados: tipo Thalictrum (T) y tipo Ranunculus (R). Los cromosomas del tipo T tienen números básicos de $\mathrm{x}=7,8 \mathrm{o}$ 13 (por ejemplo en Thalictrum L., Aquilegia L., Hydrastis J. Ellis) y son de pequeño tamaño en relación a el tipo $\mathrm{R}$ que poseen un único número básico de $\mathrm{x}=8$ (por ejemplo en Ranunculus y Clematis L.). En el caso de Halerpestes sus especies se incluían dentro de Ranunculus, y sus cromosomas se asemejan a éstos, por lo que se encontrarían ubicados dentro del grupo $\mathrm{R}$.

Tomando como referencia el número básico $\mathrm{x}=8$, los niveles de ploidía de Halerpestes varían desde diploides hasta hexaploides (Edmadzade et al., 2010), los trabajos realizados por Lozzia y Martínez (1995) y Lozzia (2007) en R. cymbalaria $(=H$. cymbalaria $)$ citan un número cromosómico esporofítico $2 \mathrm{n}=16$, mientras que para $H$. exilis no se encontró bibliografía referida a su número cromosómico y nivel de ploidía.

El objetivo de este trabajo fue caracterizar y analizar comparativamente los cariotipos de Halerpestes cymbalaria y $H$. exilis, con el fin proveer información que contribuya al esclarecimiento de problemas taxonómicos.

\section{MATERIALES Y MÉTODOS}

Las especies analizadas pertenecen a poblaciones naturales de la provincia de Tucumán y Jujuy, las cuales fueron herborizadas y depositadas en el herbario fanerogámico de la Fundación Miguel Lillo (LIL).

Halerpestes cymbalaria: ARGENTINA. Prov. Tucumán, Dpto. Tafí del Valle, Km 80, ruta 307, 1800 m snm, 11-XII-91, Lozzia 605970 (LIL); Infiernillo, Km 87, ruta 307, 3000 m snm, 12-I-93, Lozzia 606160 (LIL); frente a la escuela El Molle, Km 91,5 ruta 307, 2850 m snm, 12-I-93, Lozzia 606161 (LIL); Río Amaicha, Km 90, ruta 307, 2900 m snm, 12-I-93, Lozzia 606162 (LIL).

Halerpestes exilis: ARGENTINA. Prov. Jujuy, Dpto. Tumbaya, 2341'38,3”S 065³8'57,8” O, 4155 m snm, 17-II-2018, Andrada 166 (LIL).

Para ambas especies, el análisis mitótico se realizó en meristemas radiculares (provenientes de diez plantas por población), pretratados en 8 hidroxiquinoleina $0,002 \mathrm{M}$ durante 6 horas a temperatura ambiente, fijadas seguidamente en solución de Farmer (alcohol etílico-acido acético 3:1) y posteriormente conservados en alcohol etílico al $70 \%$. Las raíces se colorearon en hematoxilina propiónica al $2 \%$, previa hidrólisis ácida en $\mathrm{HCl} 1 \mathrm{~N}$ durante 15 minutos a $60{ }^{\circ} \mathrm{C}$. 
Los parámetros cromosómicos fueron tomados con el programa Micromeasure 3.3 (Reeves, 2001) en siete placas metafásicas como mínimo por cada especies, las medidas analizadas fueron longitud total de los cromosomas (c); longitud del brazo largo (l) y corto (s) e índice centromérico (Ic). Los cromosomas se clasificaron de acuerdo a la metodología propuesta por Levan, Fredga, Sandberg (1964) y se calcularon las asimetrías cromosómicas de acuerdo a la metodología de Romero Zarco (1986).

Las microfotografías se tomaron con una cámara Qcolor 5 montada a un microscopio Olympus BX 43. Los idiogramas correspondientes a los cariotipos de cada especie y la edición de imágenes se realizaron con el sofware CorelDraw X3.

\section{RESULTADOS}

Ambas especies de Halerpestes analizadas fueron diploides con $2 \mathrm{n}=16$ cromosomas (Fig. 1A y C) y fórmula cariotípica de $8 \mathrm{~m}+6 \mathrm{sm}+2 \mathrm{st}$ (Fig. 1D-E).
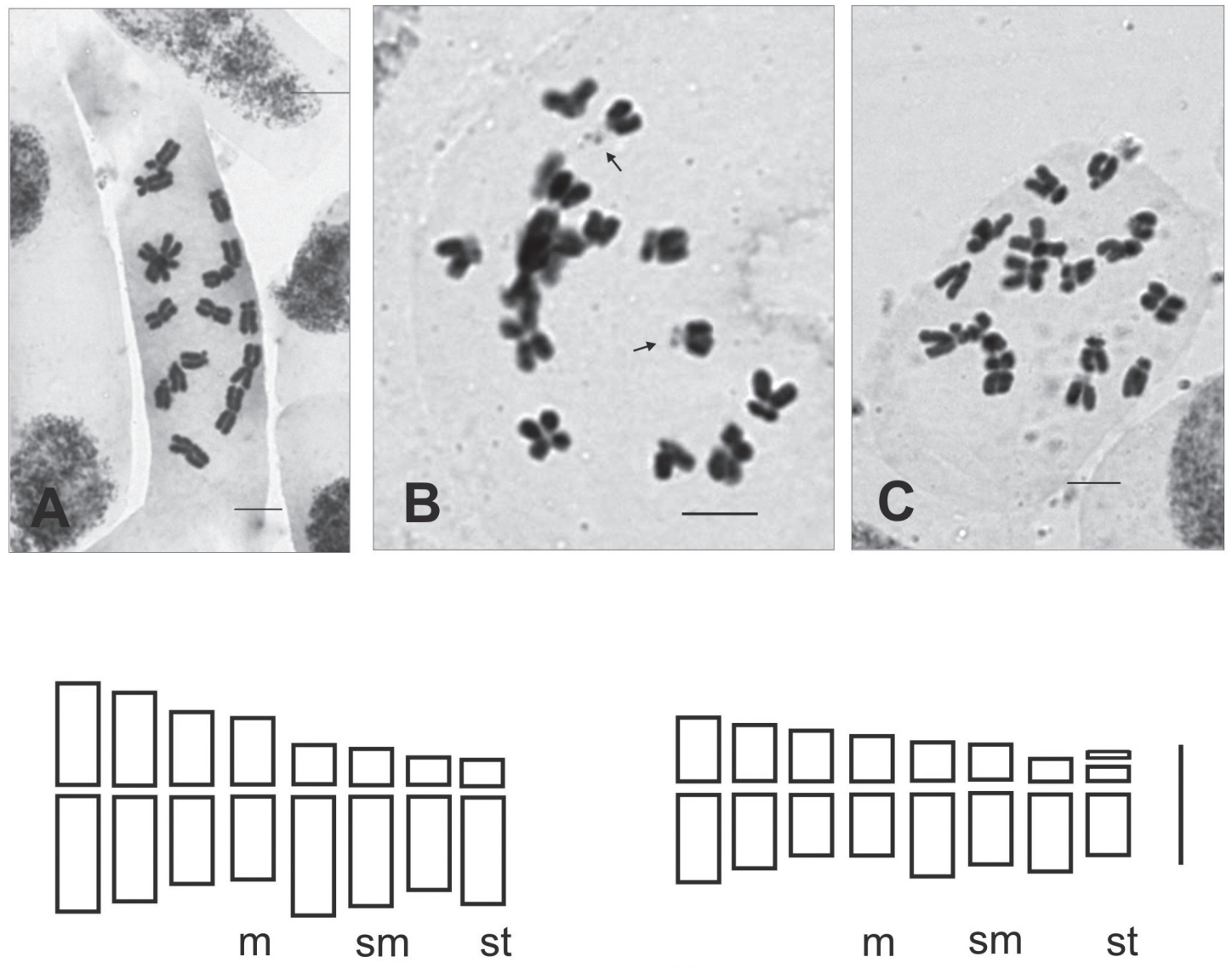

D

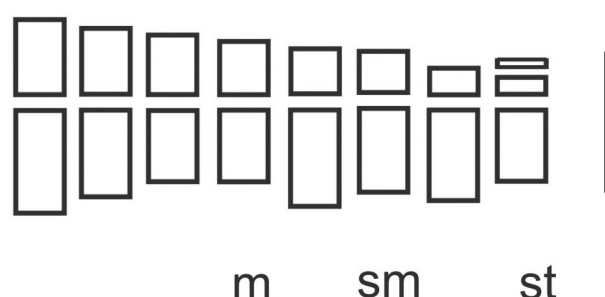

$\mathbf{E}$

Fig. 1. A-C) Metafases mitóticas. D-E) Idiogramas. A y D) Halerpestes cymbalaria. B, C, E) H. exilis (las flechas indican satélites). Escala: A, B: $5 \mu \mathrm{m} ; \mathrm{C}, \mathrm{D}: 10 \mu \mathrm{m}$. 
En Halerpestes cymbalaria los resultados fueron similares en todas las poblaciones: el rango de variación de las longitudes cromosómicas medias fueron de 3,22-5,26 $\mu \mathrm{m}$ (Tabla 1), la longitud total del complemento cromosómico haploide registrado fue de 31,09 $\mu \mathrm{m}$ y la longitud cromosómica media de 3,90 $\mu \mathrm{m}$.

En Halerpestes exilis, las longitudes cromosómicas media variaron de 1,83-3,67 $\mu \mathrm{m}$, la longitud del complemento cromosómico haploide registrado fue de 22,29 y la longitud cromosómica media fue de $2,78 \mu \mathrm{m}$. En esta especie se observó la presencia de un par de satélites sobre el brazo corto del último par cromosómico subtelocéntrico (Fig. 1B).

En ambas especies, el cariotipo es unimodal debido a la uniformidad en el tamaño cromosómico; esto se ve explicado por el valor del índice de asimetría intercromosómica $\mathrm{A}_{2}=0,20$ en Halerpestes cymbalaria y 0,19 en $H$. exilis (Tabla 2). Los

Tabla 1. Parámetros morfométricos de los cromosomas de Halerpestes cymbalaria y Halerpestes exilis. Referencias: Longitud cromosómica total media (c). Longitud media del brazo largo (I). Longitud media del brazo corto (s). Índice centromérico medio (Ic). Metacéntrico (m). Submetacéntrico (sm). Subtelocéntrico (st). Error estándar (ES).

\begin{tabular}{|c|c|c|c|c|c|c|}
\hline & $\begin{array}{c}\text { Par } \\
\text { cromosómico }\end{array}$ & $\mathrm{c}(\mu \mathrm{m}) \pm \mathrm{ES}$ & $\mathrm{I}(\mu \mathrm{m}) \pm \mathrm{ES}$ & $\mathrm{s}(\mu \mathrm{m}) \pm \mathrm{ES}$ & lc \% & $\begin{array}{c}\text { Tipo } \\
\text { cromosómico }\end{array}$ \\
\hline \multirow{8}{*}{ 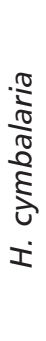 } & 1 & $5,26 \pm 0,18$ & $2,80 \pm 0,10$ & $2,46 \pm 0,11$ & 46,76 & $\mathrm{~m}$ \\
\hline & 2 & $4,78 \pm 0,22$ & $2,53 \pm 0,11$ & $2,25 \pm 0,11$ & 47,07 & $\mathrm{~m}$ \\
\hline & 3 & $3,86 \pm 0,15$ & $2,10 \pm 0,09$ & $1,76 \pm 0,08$ & 45,59 & $\mathrm{~m}$ \\
\hline & 4 & $3,62 \pm 0,16$ & $2,01 \pm 0,09$ & $1,61 \pm 0,08$ & 44,47 & $\mathrm{~m}$ \\
\hline & 5 & $3,82 \pm 0,15$ & $2,87 \pm 0,12$ & $0,95 \pm 0,07$ & 24,86 & sm \\
\hline & 6 & $3,53 \pm 0,16$ & $2,65 \pm 0,13$ & $0,88 \pm 0,06$ & 24,92 & sm \\
\hline & 7 & $2,92 \pm 0,13$ & $2,26 \pm 0,10$ & $0,66 \pm 0,05$ & 22,60 & sm \\
\hline & 8 & $3,22 \pm 0,14$ & $2,58 \pm 0,11$ & $0,64 \pm 0,05$ & 19,87 & st \\
\hline \multirow{8}{*}{$\begin{array}{l}\stackrel{n}{x} \\
\underset{0}{x} \\
\pm\end{array}$} & 1 & $3,67 \pm 0,17$ & $2,12 \pm 0,11$ & $1,55 \pm 0,08$ & 42,43 & $\mathrm{~m}$ \\
\hline & 2 & $3,15 \pm 0,17$ & $1,79 \pm 0,10$ & $1,36 \pm 0,10$ & 43,17 & $\mathrm{~m}$ \\
\hline & 3 & $2,70 \pm 0,20$ & $1,47 \pm 0,10$ & $1,23 \pm 0,10$ & 45,50 & $\mathrm{~m}$ \\
\hline & 4 & $2,58 \pm 0,19$ & $1,48 \pm 0,13$ & $1,10 \pm 0,07$ & 42,63 & $\mathrm{~m}$ \\
\hline & 5 & $2,92 \pm 0,20$ & $1,99 \pm 0,16$ & $0,93 \pm 0,07$ & 31,84 & sm \\
\hline & 6 & $2,58 \pm 0,14$ & $1,73 \pm 0,12$ & $0,85 \pm 0,05$ & 32,94 & sm \\
\hline & 7 & $2,43 \pm 0,14$ & $1,87 \pm 0,10$ & $0,55 \pm 0,05$ & 22,77 & sm \\
\hline & 8 & $1,83 \pm 0,23$ & $1,47 \pm 0,18$ & $0,35 \pm 0,06$ & 19,59 & st \\
\hline
\end{tabular}

Tabla 2. Parámetros cariotípicos de Halerpestes cymbalaria y H. exilis. Referencias: Longitud total del complemento haploide (TCL), longitud cromosómica media (c), longitud cromosómica máxima y mínima (c max., c min.), índice de asimetría intracromósomica $\left(A_{1}\right)$, índice de asimetría intercromosómica $\left(A_{2}\right)$.

\begin{tabular}{lcc}
\hline Parámetros & H. cymbalaria & H. exilis \\
\hline $2 \mathrm{n}$ & 16 & 16 \\
\hline Formula cariotípica & $8 \mathrm{~m}+6 \mathrm{sm}+2 \mathrm{st}$ & $8 \mathrm{~m}+6 \mathrm{sm}+2 \mathrm{st}$ \\
\hline $\mathrm{TCL}(\mu \mathrm{m})$ & 31,09 & 22,29 \\
\hline $\mathrm{c}(\mu \mathrm{m})$ & 3,90 & 2,78 \\
\hline $\mathrm{c} \max .(\mu \mathrm{m})$ & 5,42 & 3,84 \\
\hline $\mathrm{c} \min .(\mu \mathrm{m})$ & 2,91 & 1,26 \\
\hline $\mathrm{A}_{1}$ & 0,42 & 0,41 \\
\hline $\mathrm{A}_{2}$ & 0,20 & 0,19 \\
\hline
\end{tabular}


índice de asimetría intracromosómica $\mathrm{A}_{1}=0,42$ y 0,41 en $H$. cymbalaria y $H$ exilis respectivamente, indican cariotipos con asimetría leve.

\section{DISCUSIÓN}

Basados en el número básico de $\mathrm{x}=8$, Halerpestes cymbalaria y $H$. exilis, son taxones diploides, con $2 \mathrm{n}=16$ cromosomas y por su tamaño, los cromosomas de ambos se ubican en la categoría R (Langlet, 1932; Gregory, 1941).

Otras investigaciones reportaron recuentos diploides para Ranunculus cymbalaria (sinónimo de Halerpestes cymbalaria) (Kappor y Löve, 1970; Goepfert, 1974; Lozzia y Martínez, 1995; Lozzia, 2007; IPCN «Chromosome reports») pero no se registró información citogenética para $H$. exilis, por lo que los datos cromosómicos aportados aquí serían los primeros para la especie.

La fórmula cariotípica propuesta para $H$. cymbalaria $(=R$. cymbalaria $)$ coincide con los estudios realizados por Lozzia (2007), quien reportó longitudes cromosómicas de 2,88-5,75 $\mu \mathrm{m}$, valores próximos a los obtenidos en nuestros resultados. Además, la formula cariotípica determinada en este análisis es disímil a $8 \mathrm{sm}+8$ st reportada por Kapoor y Löve (1970) en ejemplares de Estados Unidos y $8 \mathrm{~m}+8$ a propuesta por Goepfert (1974) en material proveniente de Europa. En el caso de H. exilis, es la primera vez que se realizó un cariotipo para la especie.

Con respecto a la longitud total del complemento cromosómico haploide en Halerpestes cymbalaria, nuestros resultados se asemejan al valor de $30,90 \mu \mathrm{m}$ propuesto por Lozzia (2007). No obstante, estos valores no concuerdan con la longitud de 25,5 $\mu \mathrm{m}$ propuesto por Goepfert (1974).

Halerpestes cymbalaria y $H$. exilis se consideraron sinónimos $(=R$. cymbalaria $)$ y sus cariotipos presentaron igual fórmula, aunque existen diferencias estructurales entre los cromosomas de ambas especies: $H$. exilis exhibe satélites en el par cromosómico subtelocéntrico más pequeño que no fueron observados en $H$. cymbalaria . Así mismo, el complemento cromosómico haploide de $H$. exilis es relativamente de menor longitud que el de $H$. cymbalaria.

La asimetría en el cariotipo similares a Halerpestes exilis y $H$. cymbalaria $(=R$. cymbalaria) fueron observadas en otras especies de Ranunculus, con índices de asimetría $\mathrm{A}_{1}$ que oscilan entre 0,43-0,57; por ejemplo: $R$. apiifolius Pers., $R$. cymbalaria, $R$. flagelliformis Smith, $R$. parviflorus L., $R$. psychrophilus Wedd., $R$. praemorsus $\mathrm{H}$. B. K. ex DC., $R$. repens L. y $R$. sarmentosus Griseb (Kapoor y Löve, 1970; Lozzia, 2007). Este último autor relacionó esta asimetría con la especialización morfológica de las plantas y menciona que la familia Ranunculaceae y el género Ranunculus fueron clasificados como grupos antiguos de plantas en base a sus carpelos libres, sus partes florales ordenadas en espiral, apocarpia, carpelos cerrados imperfectamente y folículos. Lozzia (2007) también argumentó que la presencia de asimetría en los cariotipos de Ranunculaceae (incluido el género Ranunculus) hace suponer que en tiempos pasados sus complementos cromosómicos podrían haber «evolucionado» sufriendo reestructuración en la cromatina, especializándose y modificando sus ca- 
riotipos. Sin embargo, todavía persisten algunos caracteres morfológicos ancestrales en los taxones actuales, como los mencionados con anterioridad.

Kiesling (1994) mencionó que Halerpestes cymbalaria (Ranunculus cymbalaria) presenta la hojas de morfología muy variada, con lámina suborbicular, ovada, oblonga, con bordes crenados o trilobadas. Asímismo, el hecho de que nuestra fórmula cariotípica no coincida con la de Kapoor y Löve (1970) y Goepfert (1974) para esta especie, evidencia una restructuración de la cromatina entre los brazos de los cromosomas, que condujeron a las diferencias observadas entre sus cariotipos. Tebbitt y Andrada (2017) estudiaron poblaciones naturales del género Begonia L. en el Noroeste Argentino (NOA) y observaron que B. boliviensis C.DC. se caracterizaba por poseer una gran variedad morfológica: sus delgadas hojas se ensanchaban (así como sus pétalos) y presentaban bordes foliares con dientes más pronunciados a medida avanzaban hacia el norte, desde Tucumán hacia Jujuy. Los autores concluyendo que había flujo génico entre esta $B$. boliviensis y $B$. micranthera Griseb., un taxón con el que comparten el territorio y que tiene hojas cordiformes, tépalos ovados u obovados. Los investigadores sugirieron que los híbridos originales entre estas dos especies se habrían cruzado nuevamente con $B$. boliviensis, reemplazando la mayor parte de los genes de $B$. micranthera, adquiriendo con el tiempo una morfología más afín a la primera especie. Estas observaciones podrían explicar lo que ocurre con $H$. cymbalaria, posiblemente entre el material analizado por los investigadores precedentes se encontraban muestras no puras, con flujo génico interespecífico entre $H$. cymbalaria y taxones afines. El flujo génico podría ser el responsable de las diferencias encontradas en las fórmulas cariotípicas y morfología foliar de esta planta cosmopolita.

\section{CONCLUSIONES}

En la presente contribución se determinó que ambos taxones estudiados son diploides. Por primera vez se menciona recuentos cromosómico y formula cariotípica de Halerpestes exilis. Además, se establece que a pesar de que $H$. cymbalaria y $H$. exilis presentan formulas cariotípica semejantes, las diferencias estructurales entre sus cromosomas afianzan la actual ubicación taxonómica de ambos taxones como especies diferentes dentro del género Halerpestes.

\section{BIBLIOGRAFÍA / BIBLIOGRAPHY}

Emadzade, K., Lehnebach, C., Lockhart, P. y Hörandl, E. (2010). A molecular phylogeny, morphology and classification of genera of Ranunculeae (Ranunculaceae). Taxon 59 (3): 809-828. doi: 10.2307/25677670

Gregory, W. C. (1941). Phylogenetic and cytological studies in the Ranunculaceae Juss. Transactions of the American Philosophical Society New series. 31: 443-521.

Greene, E. L. (1899). Studies in the Cruciferae III. In: Pittonia 4: 187-211. The Law Reporter Company Press. Washington D.C. 
Golbatt, P. y Johnson, D. E. (1979). Index to plant chromosome numbers. Golbatt, P y Johnson, D. E. (Eds.) Missouri Botanical Garden, St. Louis, USA.

Goepfert, D. (1974). Karyotypes and DNA content in species of Ranunculus L. and related genera. Botaniska Notiser 127: 464-489.

Kapoor, B. M. y Löve, Ä. (1970). Chromosomes of Rocky Mountain Ranunculus, Caryologia 23 (4): 575-594. doi: 10.1080/00087114.1970.10796396

Kiesling, R. (1994). Ranunculaceae. En: Flora de San Juan. República Argentina. Volumen I. Vazquez Mazzini (Eds.) Buenos Aires, Argentina.

Langlet, O. 1932. Über chromosenverhaltnisse und systematik der Ranunculaceae. Svensk Botanisk Tidskrift 26: 381-400.

Levan, A., Fredga, K. L. y Sandberg, A. A. (1964). Nomenclature for centromeric position on chromosomes. Hereditas 52: 201-220. doi: 10.1111/j.1601-5223.1964. tb01953.x

Lewis, W. H., Suda Y. y Oliver R. L. (1967). In Chromosome numbers of phanerogams. 2. Annals of the Missouri Botanical Garden 54: 178-181.

Lourteig, A. (1951). Ranunculáceas de Sudamérica templada. Darwiniana 9: $397-$ 608.

Lozzia, M. E. y Martínez, A. J. (1995). Recuentos cromosómicos en especies del Género Ranunculus L. de la Provincia de Tucumán. Lilloa 38 (2): 179.

Lozzia, M. E. (2007). Estudios citogenéticos en especies del género Ranunculus de la provincia de Tucumán. (Tesis Doctoral), Universidad Nacional de Tucumán. Argentina.

Reeves, A. (2001). MicroMeasure: a new computer program for the collection and analysis of cytogenetic data. Genome 44: 439-443. doi:10.1139/g01-037

Romero Zarco, C. (1986). A new method for estimating karyotype asymmetry. Taxon 3: 531-536. doi: 10.2307/1221906

Tamura, M. (1995). Ranunculaceae. In P. Hiepko (ed), Die Natürlichen Pflanzenfamilien. Aufl. II. 17a (4): 1-555. Berlin. Duncker \& Humbolt.

Tebbitt, M. C. y Andrada, A. R. (2017). A new hybrid tuberous Begonia from Argentina. The Begonian 84: 179-184.

Ziman, S. y Keener, C. S. (1989). A geographical analysis of the family Ranuculaceae. Annals of Missouri Botanical Garden 76: 1012-1049. doi: 10.2307/2399690

Zuloaga, F. O. y Morrone, O. (1999). Catálogo de las plantas vasculares de la República Argentina. II. Dicotyledoneae. Monographs in Systematic Botany Missouri Botanical Garden 74: 1-1269.

Zuloaga, F. O., Morrone, O. y Belgrano, M. J. (2008). Catálogo de plantas vasculares del Cono Sur Volumen 3. Monographs in systematic Botany from the Missouri Botanical Garden. St. Louis, Missouri. USA. 